\title{
Ultra-Broad Band Radar Cross Section Reduction of Waveguide Slot Antenna with Metamaterials
}

\author{
Qiang $F U^{1}$, Cheng-Li $F A N^{1}$, Si-Jia $L I^{2}$, Gang $W A N G^{1}$, Xiang-Yu $C A O^{2}$ \\ ${ }^{1}$ School of Air Missile Defense, Air Force Engineering University, Xi'an 710051, China \\ ${ }^{2}$ Information and Navigation College, Air Force Engineering University, No.1 FengHao Road, Xi'an 710077, China
}

fuqiang_66688@163.com, ffq516@163.com,1sj051@126.com, iamwg@sina.com,gjgj9694@163.com

Manuscript received October 2, 2015

\begin{abstract}
To reduce the radar cross section of the waveguide slot antenna, a three-layer metamaterial is presented based on orthogonal double split-ring resonators. The absorption characteristics of three-layer metamaterial are demonstrated by simulation. Moreover, the metamaterials have been loaded on common waveguide slot antenna according to the surface current distribution. The ultrabroad band radar cross section reduction of the antenna with metamaterials had been theoretically and experimentally investigated by radiating and scattering performances. Experimental and simulated results showed that the proposed antenna with metamaterials performed broadband radar cross section reduction from $3.9 \mathrm{GHz}$ to $18 \mathrm{GHz}$ and the gain had been improved due to the coupling effect between slot and the period metamaterials structure. The maximal radar cross section reduction of the novel antenna achieved $17.81 \mathrm{~dB}$ at $8.68 \mathrm{GHz}$ for x-polarized incidence and $21.79 \mathrm{~dB}$ at $6.25 \mathrm{GHz}$ for y-polarized waves.
\end{abstract}

\section{Keywords}

Ultra-broad band, radar cross section reduction, metamaterial, gain enhancement

\section{Introduction}

It is well known that the radar cross section (RCS) of antennas for out-of-band frequencies can be significantly reduced by placing the periodic resistive surface or suitably shaped band-pass radome [1], [2]. Recently, metamaterials which have been designed and used as the reflected or radiated ground for a composition of antenna have been proposed in RCS reduction. As an effective method, the electromagnetic band-gap (EBG) loaded on ridged waveguide slot antenna array has been investigated to reduce RCS over a wide frequency range [3]. Moreover, as application of principle of passive cancellation for electromagnetic (EM) wave, the artificial magnetic conductor (AMC) and perfect electric conductor (PEC) surfaces or two AMCs were combined together for RCS reduction of waveguide slot antenna [4]. Then, two AMCs with different metamaterial structures were theoretically analyzed and easily implemented using the common printed circuit board fabrication methods for ultra-thin and broadband radar absorber material design [1], [5-7]. Nevertheless, in those cases, the RCS is reduced in boresight direction but increased in other directions.

Perfect metamaterial absorber (PMA) with ultrathin structure and near-unity absorptivity was firstly proposed and demonstrated by Landy et. al. in [8], which has become an important aspect in the research of metamaterials. Later, to achieve the polarization-insensitive, wide incident angle, broadband and multi-band absorption, many researchers made lots of efforts on the PMAs [9-13]. In 2013, a perfect metamaterial absorber with wide-angle absorption and polarization-insensitive was presented for in-band RCS reduction of waveguide slot antenna in [14]. The maximum absorptivity of $99.8 \%$ and a full width at half maximum (FWHM) of $220 \mathrm{MHz}$ could be achieved for the absorber. The broadband absorbers with different structures had been proposed based on the three layers structure in our papers [15-17]. On these bases, a novel three-layer metamaterial is proposed and applied to ultra-broad band RCS reduction of the waveguide slot antenna according to its surface current distribution. The ultra-broad band RCS reduction performance has been carefully investigated by taking the radiating performance into consideration. Measured results show that the proposed antenna performs ultra-broad band RCS reduction from $4.72 \mathrm{GHz}$ to $18 \mathrm{GHz}$. Details of the proposed antenna design and both theoretical and experimental results are presented in this paper.

\section{Design and Analysis}

The unit cell and its detailed optimum dimensions of the three-layer metamaterial are shown in Fig. 1(a). The metamaterial is composed of a metallic orthogonal double split-ring resonators (ODSRs) and metal ground plane separated by three lossy dielectric substrates. The metallic orthogonal double split-ring resonator-1 (ODSR-1), orthogonal double split-ring resonator-2 (ODSR-2) and a metal ground plane without patterning are etched on underside of 
three substrates respectively. The metal is copper with the conductivity of $5.8 \times 10^{7} \mathrm{~S} / \mathrm{m}$ and the thickness is $0.036 \mathrm{~mm}$. Modified_epoxy is used as the substrate with the relative permittivity of 4.2 and the loss tangent of 0.02 . The proposed metamaterial performs the perfect absorption due to the three common lossy substrates on one hand. It is the components of the EM wave, the reflected wave from different layers and the multi-refracted wave that superpose and counteract in working frequency on the other hand.

The absorption mechanism can be descripted by the illustration of simplified interference model [13]. The electromagnetic (EM) wave would be gradually absorbed by dielectric losses and superposed and counteracted according to the EM interference effect. In more direct perspective, the absorption is defined as $A=1-T-R=$ $1-\left|S_{11}\right|^{2}-\left|S_{21}\right|^{2}$. To maximize absorption $(A)$, we can minimize the reflection $\left(R, R=\left|S_{11}\right|^{2}\right)$ and the transmission $(T$, $\left.T=\left|S_{21}\right|^{2}\right)$ simultaneously at the same frequency range. It is noted that transmission is zero $\left(T=\left|S_{21}\right|^{2}=0\right)$ due to the copper ground plate without patterning on the underside of

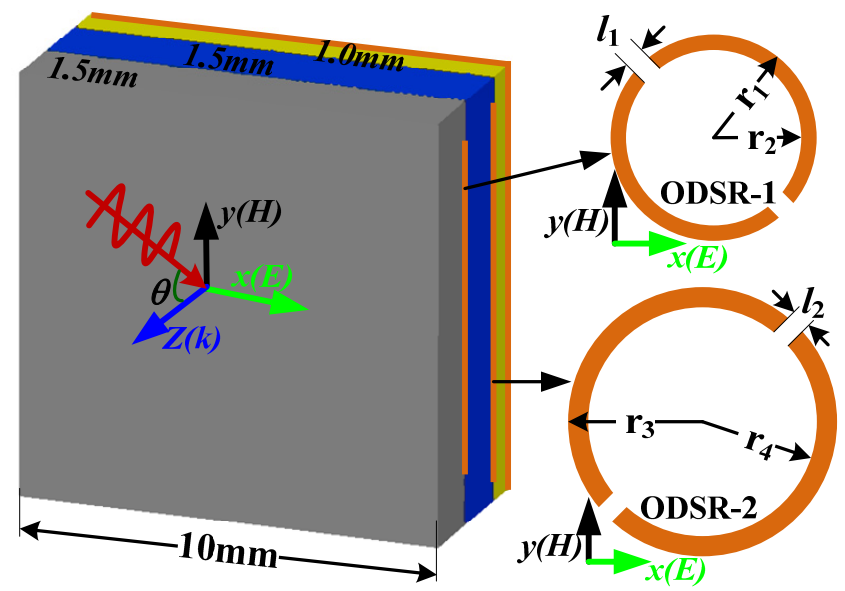

(a)

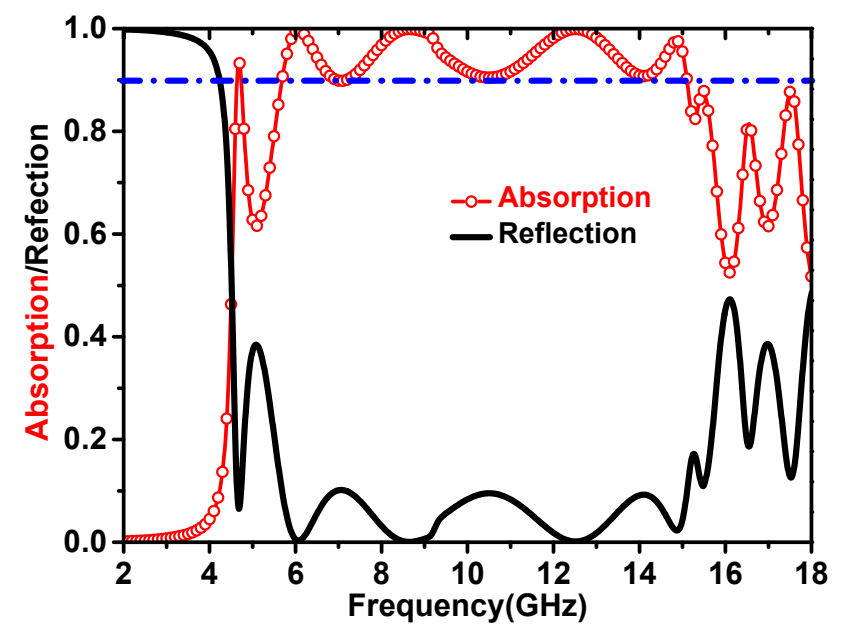

(b)

Fig. 1. Geometry of unit cell and simulated absorption and reflection results for the three-layer metamaterial. $r_{1}=3.4 \mathrm{~mm}, r_{2}=2.9 \mathrm{~mm}, r_{3}=4.6 \mathrm{~mm}, r_{4}=4.1 \mathrm{~mm}$, $w_{1}=w_{2}=0.6 \mathrm{~mm}$. (a) Geometry of metamaterial unit cell. (b) Simulated absorption and reflection results. bottom layer substrate. Then $A$ can be simplified by $A=$ $1-R=1-\left|S_{11}\right|^{2}$. It is obvious that the absorption is near $100 \%$ when the reflection is close to zero. The simulated absorption and reflection results are given in Fig. 1(b). We observe that the frequency range with absorptivity larger than $90 \%$ varies from $5.66 \mathrm{GHz}$ to $15.10 \mathrm{GHz}$ and it performs multi-absorption peaks at $4.75,6.06,8.72,12.52$, and $14.86 \mathrm{GHz}$.

To reduce RCS of the common waveguide slot antenna (common antenna), the three-layer metamaterial has been loaded on the antenna according to surface current distributions at $5.65 \mathrm{GHz}$. From Fig. 2(b), the common antenna ground has been divided into the strong current area which is on the edge of the ground and the weak current area at the center of the ground and covered by a circle with radius of $17 \mathrm{~mm}$. The metal circular surface with strong current is retained to avoid the radiation deterioration, and the metamaterials are only loaded on the weak current area for RCS reduction [15]. The metamaterial array is used as the ground of waveguide slot antenna as shown in Fig. 3. The element of " 0 " and their array are shown in Fig. 3(a). The novel waveguide slot antenna with metamaterial array given in Fig. 3(b) are designed, fabricated and measured in our paper.

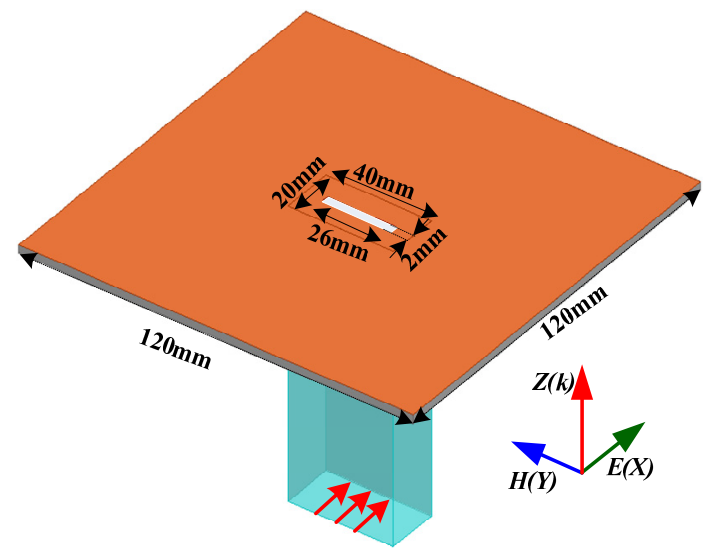

(a)

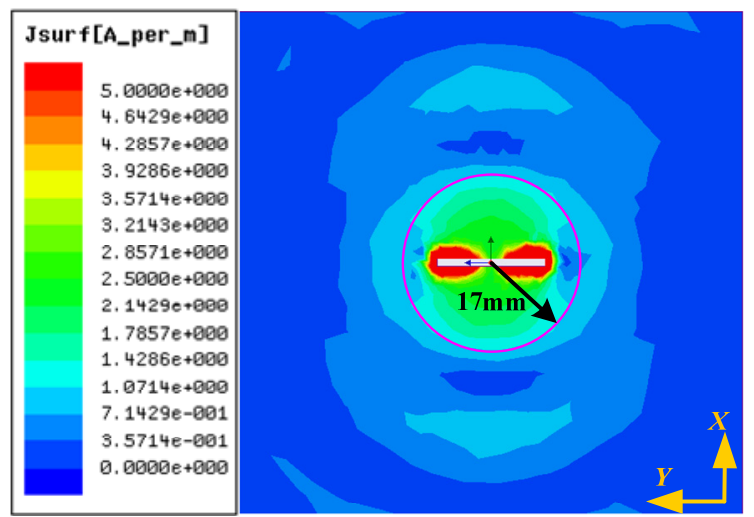

(b)

Fig. 2. Geometry and surface current distributions of the common waveguide slot antenna. (a) Geometry of common waveguide slot antenna. (b) Surface current distributions of common antenna at $5.65 \mathrm{GHz}$. 

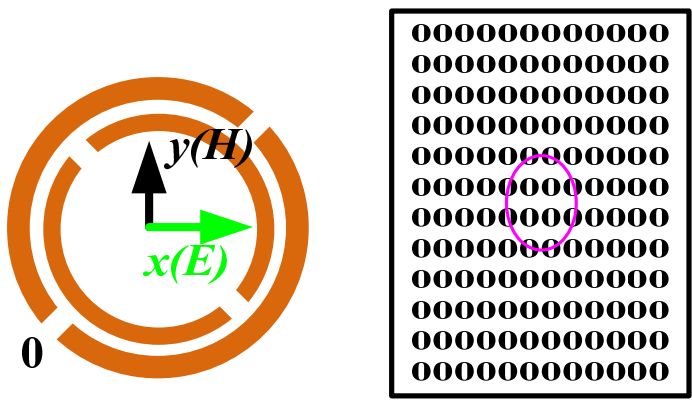

(a)

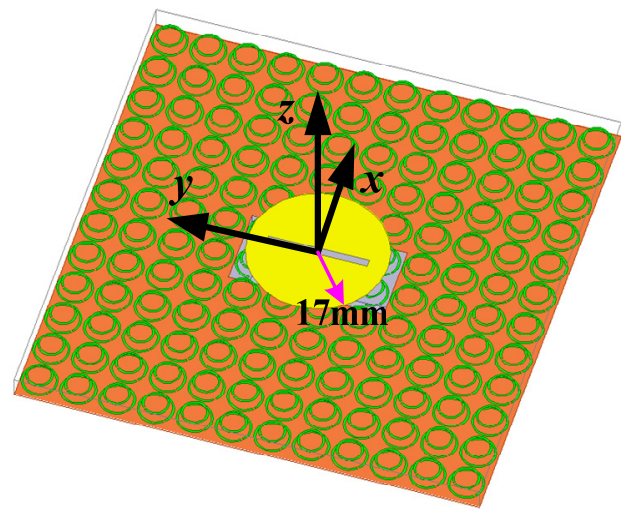

(b)

Fig. 3. Metamaterial and the waveguide slot antenna with metamaterials. (a) Metamaterial array and metamaterial cell " 0 ". (b) Waveguide slot antenna with metamaterials.

\section{Simulation and Comparison}

The RCS of the common antenna and the antenna with metamaterial array were simulated and optimized using HFSS 14.0. Simulated results of radiating characters for common antenna and the antenna with metamaterials are illustrated in Fig. 4 and 5. From Fig. 4, it can be seen that the slightly better match and wider bandwidth are performed for waveguide slot antenna with metamaterials. Simulated impedance bandwidth is from $5.58 \mathrm{GHz}$ to $5.82 \mathrm{GHz}$ with $S_{11}<-10 \mathrm{~dB}$ for the proposed antenna and from $5.48 \mathrm{GHz}$ to $5.86 \mathrm{GHz}$ for the common antenna. The slight difference between the common antenna and the antenna with metamaterial array is attributed to the coupling effects between the metamaterial array and the slot of the antenna. Simulated radiation patterns of the antenna with metamaterial array and the common antenna at $5.65 \mathrm{GHz}$ are given in Fig. 5. From Tab. 1 and Fig. 5, the beamwidth of $\mathrm{E}$ and $\mathrm{H}$ plane for the common antenna is 156 and 62 deg and that for metamaterial antenna is 80 and $57 \mathrm{deg}$. The gain of the common and proposed antenna is 6.06 and $8.27 \mathrm{dBi}$. It is clear that the gain improvement is obtained for metamaterial antenna and the $3-\mathrm{dB}$ beam widths of E-plane and H-plane are decreased at $5.65 \mathrm{GHz}$. The gain enhancement is resulted from the additional radiation components fed with metamaterial and coupling effect between slot and the period metamaterials structure. This phenomenon can be explained by the surface current dis-

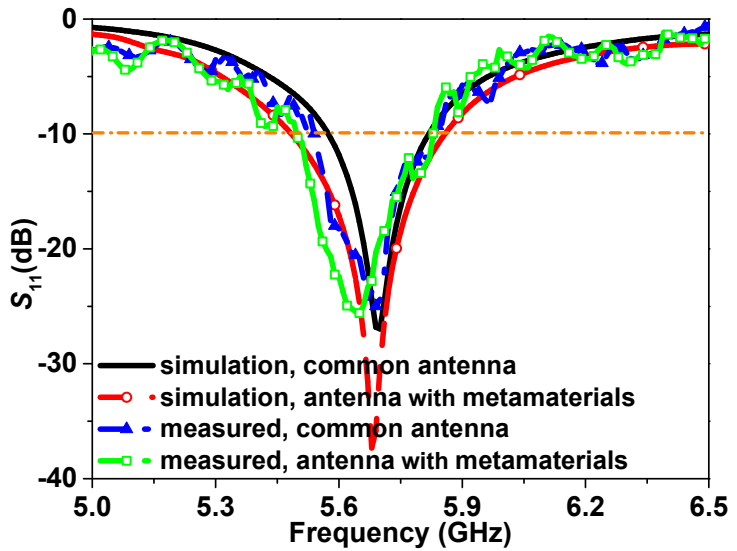

Fig. 4. Simulated and measured $S_{11}$ results of common antenna and the antenna with metamaterials.

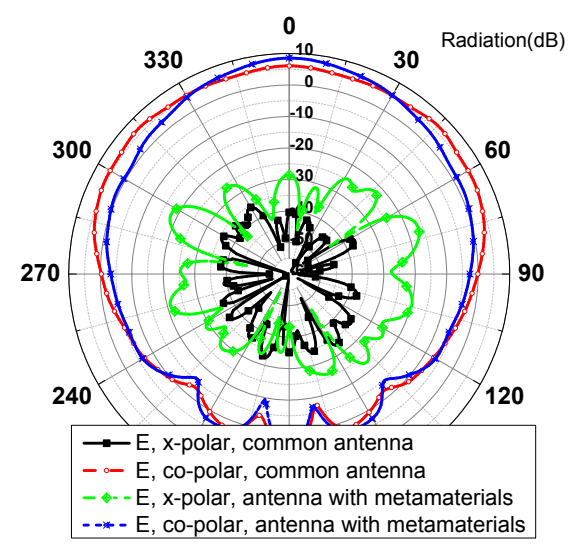

(a)

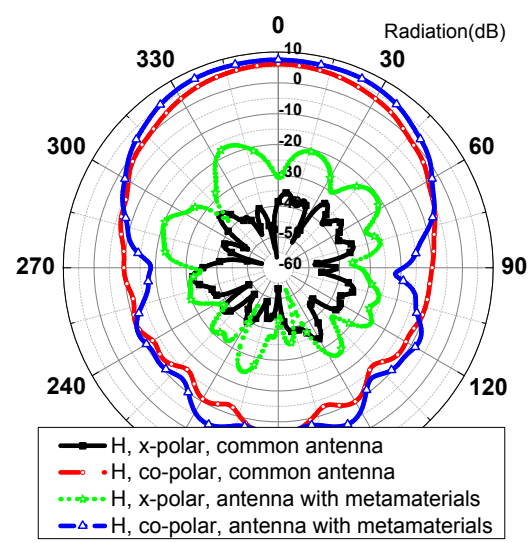

(b)

Fig. 5. Simulated radiation patterns of (a) E plane and (b) $\mathrm{H}$ plane at $5.65 \mathrm{GHz}$ of the common antenna and the antenna with metamaterials.

tributions as shown in Fig. 2(b) and Fig. 6. From Fig. 2, the surface current density on the edge of common antenna is weak and the strong surface current density is fastened on the center of the slot. From Fig. 6, the strong surface current density is not only on the center of the slot but also on the metallic orthogonal double split-ring resonators. Compared with the common waveguide slot antenna, the surface current density of antenna with metamaterials is enhanced due to the coupling effect between the slot and the period structure. 


\begin{tabular}{|c|c|c|c|}
\hline & $\begin{array}{c}\text { Frequency } \\
(\mathrm{GHz})\end{array}$ & Gain $(\mathrm{dBi})$ & $\begin{array}{c}\text { Beamwidth, E/H } \\
\text { plane (deg) }\end{array}$ \\
\hline common antenna & $5.58 \sim 5.82$ & 6.06 & $156 / 62$ \\
\hline antenna with meta. & $5.48 \sim 5.86$ & 8.27 & $80 / 57$ \\
\hline
\end{tabular}

Tab. 1. Simulated characters of the common antenna and the antenna with metamaterials.

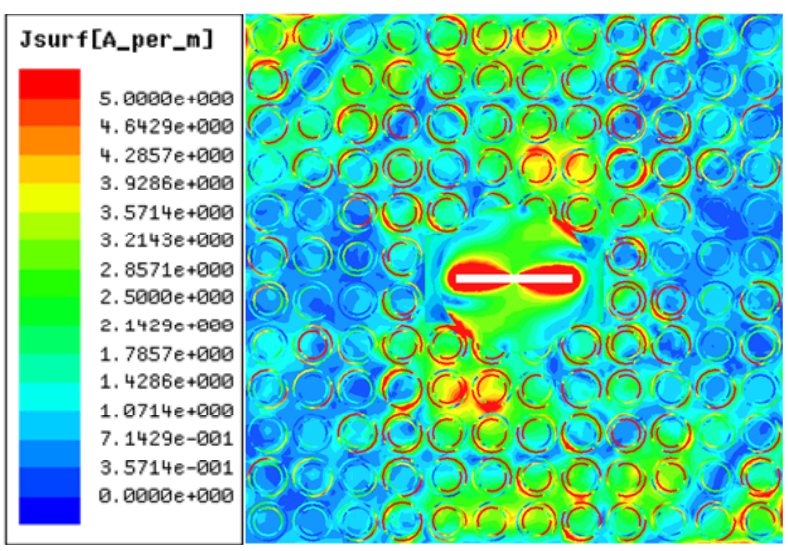

Fig. 6. Surface current distribution of the antenna with metamaterials at $5.65 \mathrm{GHz}$

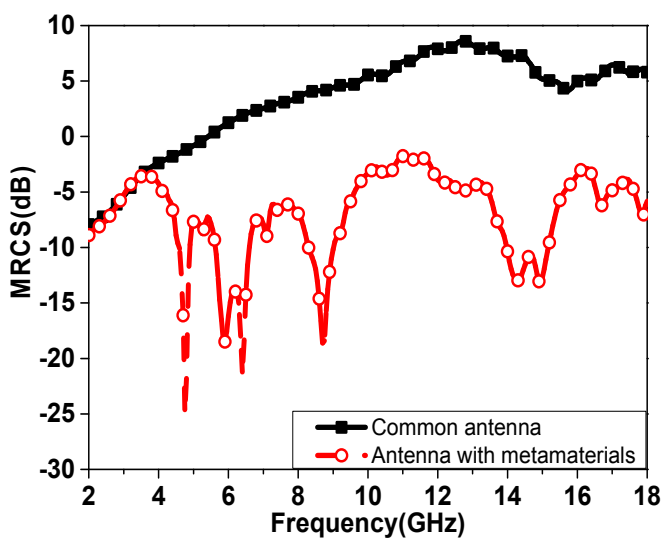

(a)

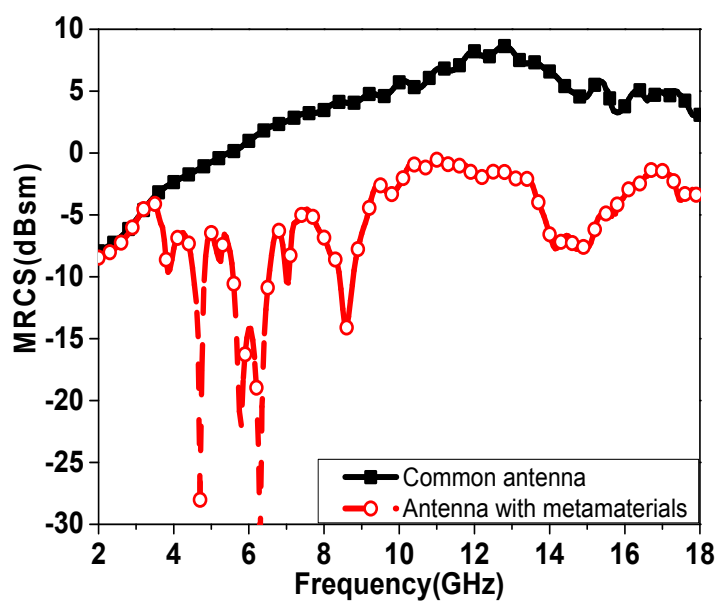

(b)

Fig. 7. Simulated results of MRCS for the common antenna and the antenna with metamaterials. (a) MRCS with xpolarized incidence. (b) MRCS with y-polarized incidence.
From Fig. 5(a) and 5(b), the gain is enhanced and the 3-dB beam widths of E-plane and H-plane are decreased at $5.65 \mathrm{GHz}$. Meanwhile, it is necessary to point out that the cross-polarization components of the antenna with metamaterial array are increased for the antenna with metamaterials owing to the coupling effects between the metamaterial array and the slot.

From Fig. 7, it is clear that the antenna with metamaterials exhibits ultra-wide band monostatic radar cross section (MRCS) reduction from $4.35 \mathrm{GHz}$ to $18 \mathrm{GHz}$ for $\mathrm{x}$ polarized incidence and from $4.22 \mathrm{GHz}$ to $18 \mathrm{GHz}$ for $\mathrm{y}$ polarized incidence, which is attributed to the broadband absorption for the three-layer metamaterial. There is a good match between the absorption bandwidth in Fig. 1(b) and the RCS reduction in Fig. 7. We note that the slight difference of ultra-wide band RCS reduction for x-polarized and $y$-polarized incidences is contributed to the polarization of the waveguide slot antenna and the asymmetry of antenna geometry.

\section{Fabrication and Measurement}

The feasibility of the proposed antenna with the metamaterial array is demonstrated in this section. The prototypes of common and metamaterial antenna have been fabricated using a common printed circuit board method on the Modified_epoxy substrates with thicknesses of $1 \mathrm{~mm}$ and $1.5 \mathrm{~mm}$ and they are illustrated intuitively in Fig. 8 . A vector network analyzer (Agilent N5230C) and two linearly polarized standard-gain horn antennas, which cover $1 \sim 18 \mathrm{GHz}$, were used to transmit and receive the EM waves for measurement. The prototypes were placed vertically at the center of a turntable to ensure that the EM wave could be similar to a plane wave on the front of prototype [18], [19]. The distance between two horn antennas and prototypes under test satisfied the far-field condition.

The measured results show that the novel antenna with metamaterial array achieves $5.8 \%(5.49 \mathrm{GHz}$ to $5.82 \mathrm{GHz})$ and common antenna obtains $4.8 \%(5.54 \mathrm{GHz}$ to $5.81 \mathrm{GHz}$ ) of impedance bandwidth. The experimental impedance bandwidth has slightly shifted due to the fabricated and experimental tolerance. The experimental radiation patterns of E- and H-planes are shown in Fig. 9. The 3 -dB beam width is suppressed from $142 \mathrm{deg}$ for common

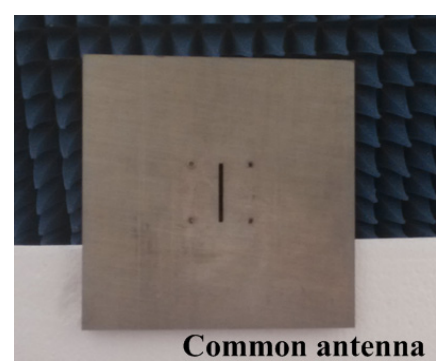

(a)

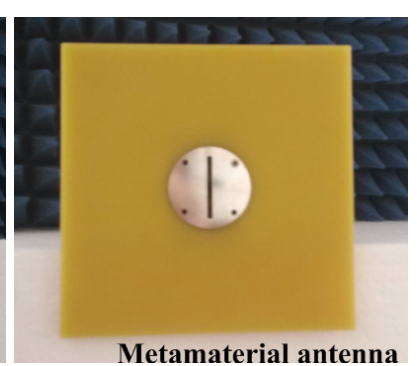

(b)
Fig. 8. Photographs of (a) common and (b) metamaterial antennas. 
antenna to $56 \mathrm{deg}$ for the novel antenna with metamaterial array in E-plane. The beamwidth of H-plane is decreased from $54 \mathrm{deg}$ to $48 \mathrm{deg}$. The measured MRCS reductions are shown in Fig. 10. It is observed that the ultra-wide band MRCS reduction can be obtained for the novel antenna with metamaterial array. The metamaterial antenna performs ultra-wide band MRCS reductions of $116.9 \%$ (4.72 GHz-18 GHz) for x-polarized incidence and 120.4\% $(4.46 \mathrm{GHz}-12 \mathrm{GHz})$ for y-polarized waves. The maximal MRCS reduction of $18.4 \mathrm{~dB}$ at $8.7 \mathrm{GHz}$ for $\mathrm{x}$-polarized incidence and $22.5 \mathrm{~dB}$ at $6.3 \mathrm{GHz}$ for y-polarized waves can be achieved from experimental results. A good agreement is obtained by experimental and simulated results.

The comparison of the proposed waveguide slot antenna with metamaterials and other antennas in references [5], [14], [18] are given in Tab. 2. It is obvious that the gain of the proposed antenna and the gain of the antennas in references [5], [18] are improved compared with the common waveguide slot antenna. Furthermore, the bandwidth of RCS reduction of $5 \mathrm{~dB}$ for the proposed antenna is the broadest of all. Moreover, the proposed antenna with metamaterial array performs ultra-broad band absorption and gain-enhancement.

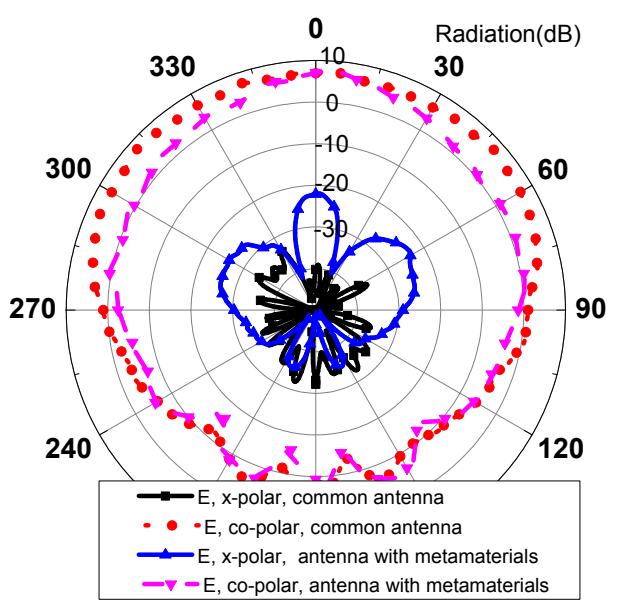

(a)

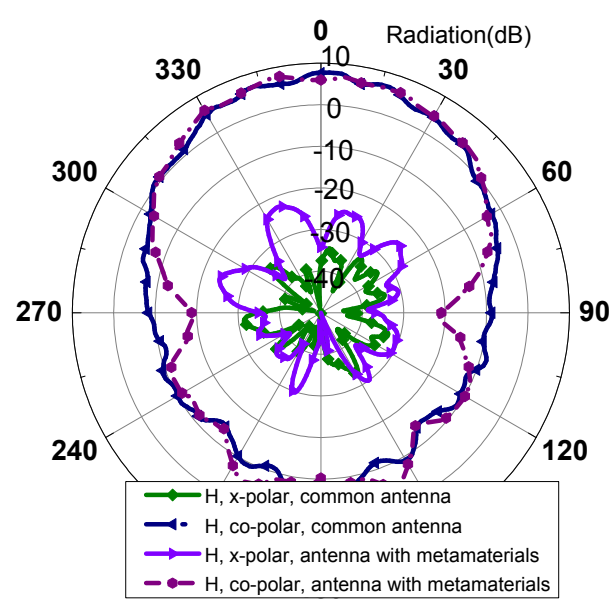

(b)

Fig. 9. Experimental radiation patterns of (a) E plane and (b) $\mathrm{H}$ plane at $5.65 \mathrm{GHz}$ of the common antenna and the proposed antenna with metamaterial array.

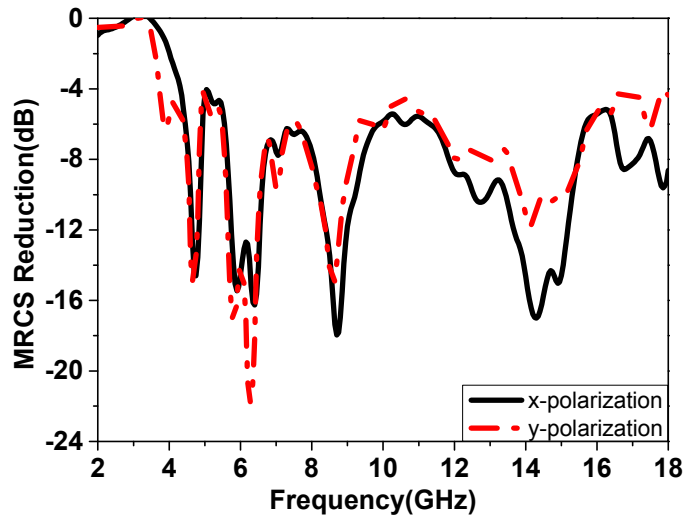

Fig. 10. The monostatic RCS reduction results between the proposed antenna and common antenna with the (a) x-polarized incident wave and (b) y-polarized incident wave.

\begin{tabular}{|c|c|c|c|}
\hline & $\begin{array}{c}\text { Relative } \\
\text { Bandwidth }\end{array}$ & $\begin{array}{c}\text { Gain Change } \\
(\mathrm{dB})\end{array}$ & $\begin{array}{c}\text { Bandwidth of RCS } \\
\text { reduction(GHz) }\end{array}$ \\
\hline $\begin{array}{c}\text { Proposed } \\
\text { antenna }\end{array}$ & $6.7 \%$ & +2.2 & $\begin{array}{c}4.7 \sim 18 \\
(\mathrm{RCSR}>5 \mathrm{~dB})\end{array}$ \\
\hline Ref. [5] & $12.2 \%$ & +3.2 & $\begin{array}{c}4.8 \sim 6.8 \\
(\mathrm{RCSR}>5 \mathrm{~dB})\end{array}$ \\
\hline Ref. [14] & $3.5 \%$ & -0.6 & $\begin{array}{c}5.5 \sim 6.2 \\
(\mathrm{RCSR}>5 \mathrm{~dB})\end{array}$ \\
\hline Ref. [18] & $7 \%$ & +1.7 & $\begin{array}{c}6.4 \sim 7.8 \\
(\mathrm{RCSR}>5 \mathrm{~dB})\end{array}$ \\
\hline
\end{tabular}

Tab. 2. Comparison of the proposed waveguide slot antenna with metamaterial and other antennas in references [5], [14], [18] ("+" means increase and "-" means decrease)

\section{Conclusion}

In this paper, an ultra-wide band RCS reduction is obtained by loading three-layer metamaterial on the common waveguide slot antenna. The metamaterials with broadband absorption are only loaded on the weak current area for RCS reduction and metal circular surface with strong current density is retained to avoid radiation deterioration. Simulated and measured results show that the proposed metamaterial waveguide slot antenna with the metamaterial array performs ultra-wide band RCS reduction not only for $x$-polarized incidence but also for y-polarized incident waves. Due to the remarkable merits of ultra wideband RCS reduction, the proposed antenna shows a potential use for the wireless military communication.

\section{Acknowledgments}

Authors thank the supports from the National Natural Science Foundation of China under Grant (No.61501494, No.61471389, and No.61271100), the Natural Science Foundational Research Fund of Shaanxi Province (No. 2012JM8003), and the Doctoral Foundation of Air Force Engineering University under Grant (No.KGD080914002). They also thank the reviewers for their valuable comments. 


\section{References}

[1] GENOVESI, S., COSTA, F., MONORCHIO, A. Wideband radar cross section reduction of slot antennas arrays. IEEE Transactions on Antennas and Propagation, 2014, vol. 62, no. 1, p. 163-173. DOI: 10.1109/TAP.2013.2287888

[2] JIA, Y. T., LIU, Y., WANG, H., LI, K., et al. Low-RCS, high-gain, and wideband mushroom antenna. IEEE Antennas and Wireless Propagation Letters, 2015, vol. 14, no. 1, p. 277-279. DOI: 10.1109/ LAWP.2014.2363071

[3] ZHANG, J. J., WANG, J. H., CHEN, M. E., et al. RCS reduction of patch array antenna by electromagnetic band-gap structure. IEEE Antennas and Wireless Propagation Letters, 2012, vol. 11, no. 1, p. 1048-1051. DOI: 10.1109/LAWP.2012.2215832

[4] PAQUAY, M., IRIARTE, J. C., EDERRA, I., et al. Thin AMC structure for radar cross-section reduction. IEEE Transactions on Antennas and Propagation, 2007, vol. 55, no. 12, p. 3630-3638. DOI: $10.1109 /$ TAP.2007.910306

[5] ZHAO, Y., CAO, X.-Y., GAO, J., et al. Broadband RCS reduction and high gain waveguide slot antenna with orthogonal array of polarisation-dependent AMC. Electronics Letters, 2013, vol. 49, no. 21 , p. 1312-1313. DOI: 10.1049/el.2013.2417

[6] IRIARTE GALARREGUI, J. C., TELlECHEA PEREDA, A., MARTINEZ DE FALCÓN, J. L., et al. Broadband radar crosssection reduction using AMC technology. IEEE Transactions on Antennas and Propagation, 2013, vol. 61, no. 12, p. 6136-6143. DOI: 10.1109 /TAP.2013.2282915

[7] LI, Y. Q., ZHANG, H., FU, Y. Q., et al. RCS reduction of ridged waveguide slot antenna array using EBG radar absorbing material. IEEE Antennas and Wireless Propagation Letters, 2008, vol. 7, no. 1, p. 473-476. DOI: 10.1109/LAWP.2008.2001548

[8] LANDY, N. I., SAJUYIGBE, S., MOCK, J. J., et al. A perfect metamaterial absorber. Physical Review Letters, 2008, vol. 100, p. 207402. DOI: 10.1103/PhysRevLett.100.207402

[9] LI, L., YANG, Y., LIANG, C. H. A wide-angle polarizationinsensitive ultra-thin metamaterial absorber with three resonant modes. Journal of Applied Physics, 2011, vol. 110, no. 6, p. 063702 . DOI: $10.1063 / 1.3638118$

[10] LI, S. J., GAO, J., CAO, X. Y., ZHANG, Z. Loaded metamaterial perfect absorber using substrate integrated cavity. Journal of Applied Physics, 2014, vol. 115, no. 21, p. 213703. DOI: $10.1063 / 1.4881115$

[11] SUN, L. K., CHENG, H. F., ZHOU, Y. J., WANG, J. Broadband metamaterial absorber based on coupling resistive frequency selective surface. Optics Express, 2012, vol. 20, no. 4, p. 4675-4680. DOI: $10.1364 /$ OE.20.004675

[12] YOO, M., LIM, S. Polarization-independent and ultra wide band metamaterial absorber using a hexagonal artificial impedance surface and a resistor-capacitor layer. IEEE Transactions on Antennas and Propagation, 2014, vol. 62, no. 5, p. 2652-2658. DOI: 10.1109/TAP.2014.2308511

[13] LI, S. J., GAO, J., CAO, X. Y., ZHANG, Z., ZHENG, Y. J., et al. Multiband and broadband polarization-insensitive perfect absorber devices based on a tunable and thin double split-ring metamaterial. Optics Express, 2015, vol. 23, no. 3, p. 3523-3533. DOI: 10.1364/OE.23. 003523

[14] LIU, T., CAO, X. Y., GAO, J., et al. RCS reduction of waveguide slot antenna with metamaterial absorber. IEEE Transactions on Antennas and Propagation, 2013, vol. 61, no. 4, p. 2327-2335, DOI: 10.11 09/TAP.2012.2231922

[15] LI, S. J., GAO, J., CAO, X. Y., et al. Loading metamaterial perfect absorber method for in-band radar cross section reduction based on the surface current distribution of array antennas. IET Microwave
Antennas and Propagation, 2015, vol. 9, no. 5, p. 399-406. DOI: 10.1049/iet-map.2014.0490

[16] LI, S. J., GAO, J., CAO, X. Y., et al. Polarization-insensitive and thin stereometamaterial with broadband angular absorption for oblique incidence. Applied Physics A, 2015, vol. 119, no. 1, p. 371-378. DOI: $10.1007 / \mathrm{s} 00339-014-8978-\mathrm{y}$

[17] LI, S. J., GAO, J., CAO, X. Y., et al. Analysis and design of three layers perfect metamaterial-inspired absorber based on double split-serration-rings structure. IEEE Transaction on Antennas and Propagation, 2015, vol. 63, no. 11, p. 5155-5160. DOI: 10.1109/TAP.2015.2475634

[18] TAN, Y., YUAN, N., YANG, Y., et al. Improved RCS and efficient waveguide slot antenna. Electronics Letters, 2011, vol. 47, no. 10 , p. 582-583. DOI: 10.1049/el.2011.0842

[19] JIANG, W., ZHANG, Y., DENG, Z. B., et al. Novel technique for RCS reduction of circularly polarized microstrip antennas. Journal of Electromagnetic Waves and Applications, 2013, vol. 27, no. 9, p. 1077-1088. DOI: 10.1080/09205071.2013.800461

\section{About the Authors ...}

Qiang FU received the B.S. degree from Sun Yat-sen University in 2010, and M.S. degree from the School of Air Missile Defense, Air Force Engineering University, China, in 2012, respectively. Currently, he is working toward his Ph.D degree at the School of Air Missile Defense, Air Force Engineering University. His research interest is in maneuvering target tracking, electromagnetic metamaterial and their applications.

Cheng-Li FAN received the B.S. degree from the Telecommunication Engineering Institute, Air Force Engineering University in 2009, and M.S. degree from the School of Air Missile Defense, Air Force Engineering University, China, in 2011, respectively. Currently, she is working toward her Ph.D degree at the School of Air Missile Defense, Air Force Engineering University. Her research interest is in intelligent information processing; information fusion, electromagnetic metamaterial and antenna applications.

Si-Jia LI was born in Xi'an, Shaanxi province, P.R. China in 1987. He received the B. Eng. degree in Electronics and Information Engineering from Guangxi University, Nanning, China, in 2009 and the M. Eng. degree in Information and Telecommunication Engineering from the Air Force Engineering University, Xi'an China, in 2011. He is currently working toward his Ph.D degree in Electronic Science and Technology at the Information and Navigation College of the Air Force Engineering University. His research activity has been focused on the broadband and fractal perfect metamaterial absorber and its application for $\mathrm{RCS}$ reduction of antennas.

Gang WANG received the B. S. and M. S. degrees from the Air Force Missile Institute in 1994 and 1997, respectively. He joined the Air Force Missile Institute in 1997 as an assistant teacher. He became an associate professor in 2005. Now, he is a professor of the Air Force Engineering University. His research interests include intelligent information processing, smart antennas, and their applications. 